\title{
Illustrated Handbook of Cardiac Surgery
}




\section{Springer}

New York

Berlin

Heidelberg

Barcelona

Budapest

Hong Kong

London

Milan

Paris

Tokyo 


\section{Illustrated Handbook of Cardiac Surgery}

Bradley J. Harlan, MD

Section of Thoracic and Cardiovascular Surgery, Sutter Memorial Hospital,

Sacramento, California; Assistant Clinical Professor of Surgery, University of California Davis Medical School, Sacramento, California; Formerly: Associate Professor of Surgery, Oregon Health Sciences University, Portland, Oregon

\section{Albert Starr, MD}

Professor of Surgery, Oregon Health Sciences University, Portland, Oregon;

Director, Heart Institute at St. Vincent Hospital, Portland, Oregon

\section{Fredric M. Harwin, BFA, MS}

Harwin Studios, Portland, Oregon 
Bradley J. Harlan, MD

5301 F Street, Suite 312

Sacramento, California 95819

USA

Fredric M. Harwin

Harwin Studios

9101 SW 15th Avenue

Portland, OR 97219

USA

With Contributions by Alain Carpentier, MD

Professor of Cardiac Surgery, Hôpital Broussais, University of Paris, Paris, France

With 266 Illustrations

Library of Congress Cataloging-in-Publication Data

Harlan, Bradley J.

Illustrated handbook of cardiac surgery / Bradley J. Harlan,

Albert Starr, Fredric M. Harwin.

p. $\mathrm{cm}$.

Includes bibliographical references and index.

ISBN-13:978-0-387-94447-0 e-ISBN-13:978-1-4612-2324-5

DOI: $10.1007 / 978-1-4612-2324-5$

1. Heart-Surgery-Handbooks, manuals, etc. I. Starr, Albert,

1926- . II. Harwin, Fredric M. III. Title.

[DNLM: 1. Cardiovascular Diseases-surgery-handbooks. WG 39

H283i 1995]

RD598.H318 1995

$617.4^{\prime} 12-\mathrm{dc} 20$

$\mathrm{DNLM} / \mathrm{DLC}$

for Library of Congress

95-179

Printed on acid-free paper.

(c) 1996 Springer-Verlag New York Inc.

All rights reserved. This work may not be translated or copied in whole or in part without the written permission of the publisher (Springer-Verlag New York, Inc., 175 Fifth Avenue, New York, NY 10010, USA), except for brief excerpts in connection with reviews or scholarly analysis. Use in connection with any form of information and retrieval, electronic adaptation, computer software, or by similar or dissimilar methodology now known or hereafter developed is forbidden.

The use of general descriptive names, trade names, trademarks, etc., in this publication, even if the former are not especially identified, is not to be taken as a sign that such names, as understood by the Trade Marks and Merchandise Marks Act, may accordingly be used freely by anyone.

While the advice and information in this book are believed to be true and accurate at the date of going to press, neither the authors nor the editors nor the publisher can accept any legal responsibility for any errors or omissions that may be made. The publisher makes no warranty, express or implied, with respect to the material contained herein.

Production coordinated by Chernow Editorial Services, Inc., and managed by Natalie Johnson; manufacturing supervised by Jacqui Ashri.

Typeset by Bytheway Typesetting Services, Norwich, NY.

987654321

ISBN-13:978-0-387-94447-0 Springer-Verlag New York Berlin Heidelberg 
To our wives, Sharon, Victoria, and Sara and to our families, whose love and support made this book possible 


\section{Preface}

The Illustrated Handbook of Cardiac Surgery is adapted from the Second Edition of the color illustrated Manual of Cardiac Surgery (Springer-Verlag, 1995). The Illustrated Handbook of Cardiac Surgery communicates all the important information, but eliminates the detailed explanation of judgment and technique that is relevant only to cardiac surgeons or other individuals intensely interested in these details. The chapters covering operations explain the basic concepts and fundamentals of the operations. A selection of the most important bibliographic sources follows each chapter, but are not cited in the text. Individuals interested in more exhaustive coverage of information sources should consult the Second Edition of the Manual of Cardiac Surgery.

The Illustrated Handbook of Cardiac Surgery should appeal to all individuals caring for cardiac surgical patients: cardiologists, anesthesiologists, radiologists, general surgery residents rotating on cardiac surgery, medical students, physician assistants, nurses, perfusionists, and all other members of the cardiac surgery team. It should even appeal to laypeople who want to read about cardiac surgery in more depth than is available in lay publications. Our attempt has been to produce a book that is clearly written, clearly illustrated, and affordable.

The techniques chosen are those developed over three decades of clinical practice and resident training at the Oregon Health Sciences University and St. Vincent Hospital, Portland, Oregon, and Sutter Memorial Hospital, Sacramento, California. These techniques have served us well.

The first author moved from the Oregon Health Sciences University to Sutter Memorial Hospital in Sacramento in 1981. During the ensuing decade some of the techniques used by the first two authors have evolved in different directions. Where this is the case, the text indicates where the technique shown is used at the present time.

The text authors are proud to have the illustrator, Mr. Fredric Harwin, as coauthor. Mr. Harwin has been a significant contributor to the basic concepts and intellectual substance of the book. His art is a powerful instrument of education.

It is hoped that this book will increase knowledge and understanding of cardiac surgery and thereby improve patient care.

Bradley J. Harlan, M.D. Albert Starr, M.D.

A medical illustrator must understand the philosophies and techniques of the scientist as well as those of the artist. For the Illustrated Handbook of Cardiac 
Surgery I worked with Dr. Harlan and Dr. Starr to create illustrations that allow the reader to visualize the surgical field as the surgeon sees it. For each illustration, I drew upon direct observation, operative photographs, fresh specimens, and, most importantly, extensive consultations with my surgeon co-authors. The sequence of creation was, first, discussion of desired illustrations, then a rough sketch, consultation with the surgeons, finished pencil drawing, another consultation, then the final rendering. These renderings were then checked against actual surgery for accuracy in representation of tissues, instrumentation, tissue responses to manipulation, and consistency of representation.

Close communication between artist and surgeon is essential to produce illustrations that are both anatomically and surgically correct and artistically viable.

Fredric M. Harwin 


\section{Acknowledgments}

We wish to thank our colleagues who directly and indirectly inspired many concepts of this book and provided support and stimulation during its creation: from Sacramento, Edward A. Smeloff, George E. Miller Jr, Paul B. Kelly, Jr, Forrest L. Junod, Kenneth A. Ross, Kuppe G. Shankar, Douglas R. Schuch, Michael T. Ingram, and Eric A. Peper, and from Portland, James A. Wood, Richard D. Chapman, Aftab Ahmad, H. Storm Floten, Jeffrey Swanson, Hugh L. Gately, Duane S. Bietz, Hagop Hovaguimian, and Anthony P. Furnary.

Valuable review and modification of the manuscript was provided by A. Glen Brooksby, Charles F. Collins, Jeffrey Leon, and Cornelius G. Wesseling for the chapter, "Anesthesia for Cardiac Surgery," and by Pat Brady and Gregory Meiling for the chapter "Conduct of Cardiopulmonary Bypass." We would like to thank Rodd Ambroson and Jeanne Koelling for their assistance with the production of illustrations. The creation of the illustrations was enhanced by the excellent photographs of our operations taken by Norman Meder. Gratitude is extended to Dee Sanchez, who helped greatly with the logistics required when a book is written and illustrated by authors living in different cities.

Finally, our thanks to Springer-Verlag, their superb staff, and our editor, William Day. We consider ourselves very fortunate to be beneficiaries of Springer-Verlag's longstanding expertise in scientific publishing and their strong commitment to quality. 


\section{Contents}

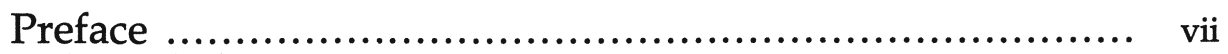

Acknowledgments ........................................ ix

1 Preoperative Preparation ............................................ 1

Routine Preparation ................................................... 1

Medication Management …...................................... 3

Patient and Family Preparation ...................................... 4

Bibliography .......................................................... 4

2 Anesthesia for Cardiac Surgery ................................... 6

Monitoring ........................................................... 6

Myocardial Protection: The Anesthesiologist's Role ................. 8

Anesthetic Agents ................................................... 9

Paralyzing Agents …............................................. 10

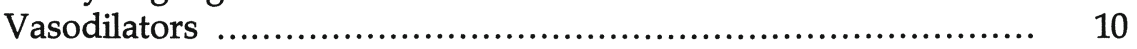

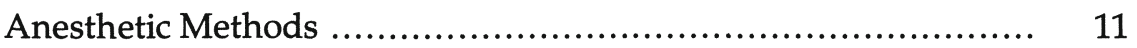

Bibliography ...................................................... 11

3 Preparation for Cardiopulmonary Bypass .................... 13

Skin Preparation and Draping ........................................... 13

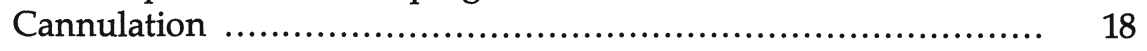

4 Conduct of Cardiopulmonary Bypass …..................... 23

Standard Bypass ......................................................... 23

Cardiopulmonary Bypass in Neonates and Children ................ 28

Bibliography ....................................................... 29

5 Myocardial Preservation .............................................. 31

Operative (Mechanical) Coronary Perfusion ......................... 31

Normothermic Ischemic Arrest ...................................... 34

Ischemia Modified by Topical Hypothermia …...................... 39

Cold Cardioplegic Arrest .............................................. 40

Warm Blood Cardioplegia ............................................ 45

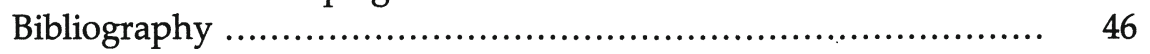

6 Postoperative Care .................................................... 49

Routine Monitoring …................................................. 49

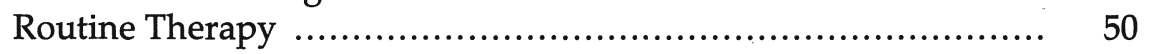

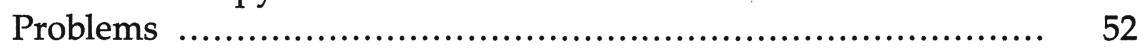

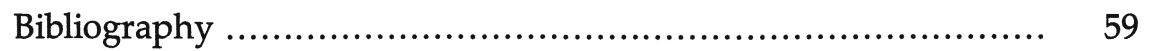


7 Low Cardiac Output: Pathophysiology

and Treatment............................................................ 61

Determinants of Cardiac Output ..................................... 61

Manipulation of Preload ............................................. 62

Manipulation of Afterload .......................................... 62

Manipulation of Myocardial Contractility ........................... 63

Indications for Intraaortic Balloon Counterpulsation ................ 64

Technique of Intraaortic Balloon Insertion ............................ 65

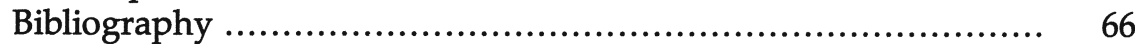

8 Coronary Artery Surgery ........................................... 68

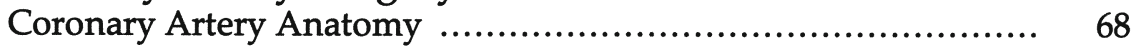

Coronary Arteriography …....................................... 72

Noninvasive Methods of Diagnosis of Coronary

Artery Disease ...................................................... 76

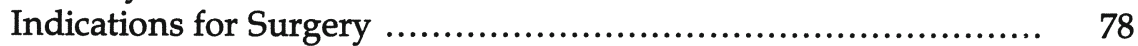

Surgical Strategy …............................................. 82

Surgical Technique ................................................. 87

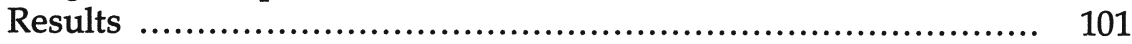

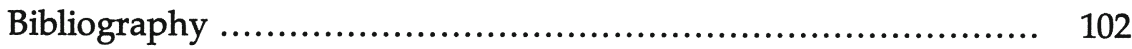

9 Left Ventricular Aneurysm .......................................... 105

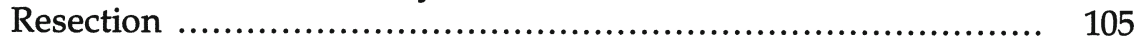

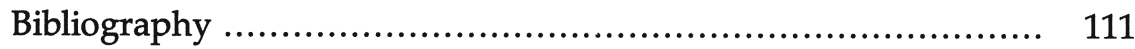

10 Postinfarction Ventricular Septal Defect ...................... 113

Indications for Surgery ............................................... 113

Surgical Strategy .................................................... 113

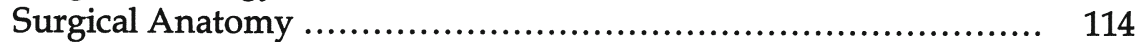

Surgical Technique …............................................. 114

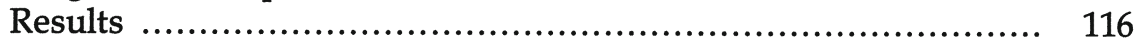

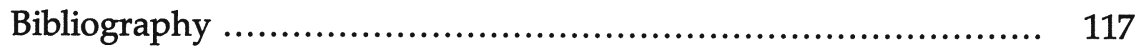

11 Mitral Valve Surgery .................................................. 118

With Alain Carpentier

Commissurotomy …............................................... 118

Valvuloplasty/Anuloplasty ....................................... 124

Replacement ......................................................... 131

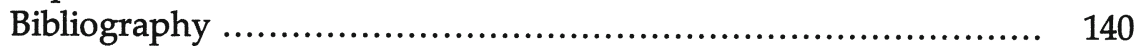

12 Aortic Valve Surgery ................................................... 143

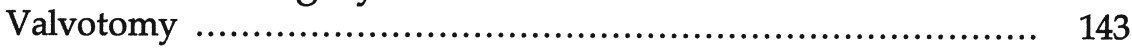

Replacement ....................................................... 145

Surgical Relief of Other Forms of Left Ventricular

Outflow Obstruction .................................................... 163

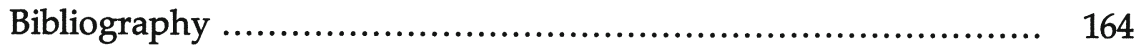

13 Tricuspid Valve Surgery ........................................ 167

With Alain Carpentier

Anuloplasty 


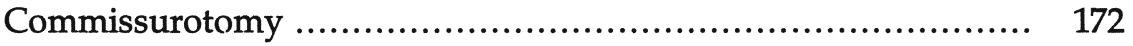

Replacement ..................................................... 173

Bibliography .................................................. 174

14 Patent Ductus Arteriosus .......................................... 175

Indications for Surgery .......................................... 175

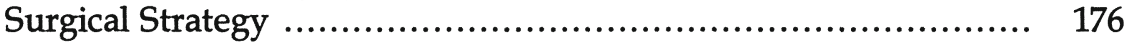

Surgical Anatomy ............................................... 177

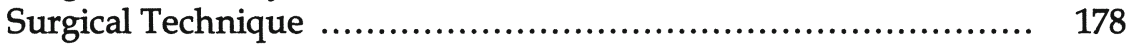

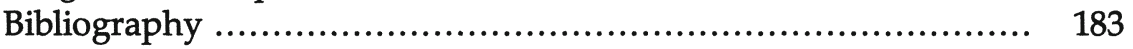

15 Coarctation of the Aorta ............................................. 184

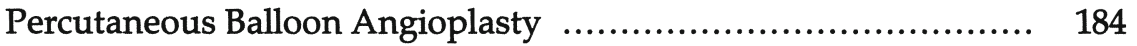

Indications for Surgery ........................................... 185

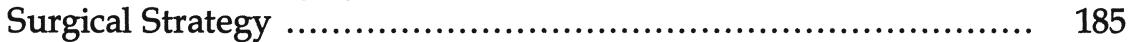

Surgical Anatomy ................................................ 187

Surgical Technique .............................................. 189

Postoperative Paradoxical Hypertension ........................... 199

Results ......................................................... 200

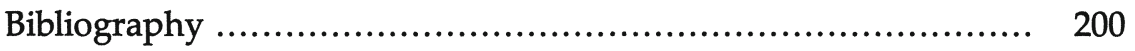

16 Systemic-Pulmonary Shunts ................................... 203

History ............................................................. 203

Classic Blalock-Taussig Shunt .................................... 204

Modified Blalock-Taussig Shunt ................................... 207

Central Shunt ..................................................... 209

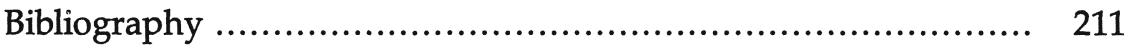

17 Pulmonary Valve Stenosis ........................................ 212

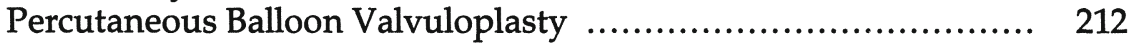

Pulmonary Valvotomy .............................................. 212

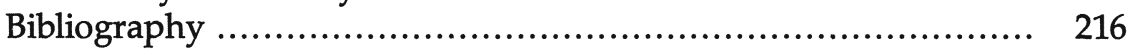

18 Atrial Septal Defects ................................................ 218

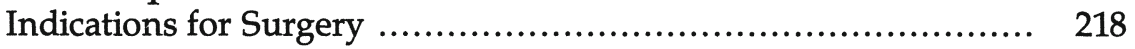

Sinus Venosus Defect ........................................ 219

Ostium Secundum Defects ........................................ 221

Ostium Primum Defect .......................................... 223

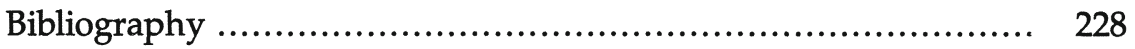

19 Complete Atrioventricular Canal ............................. 230

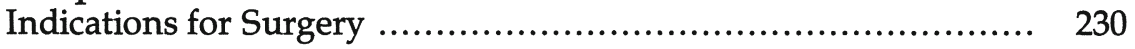

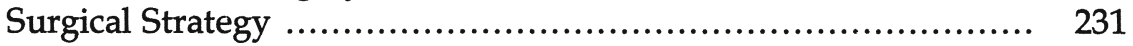

Surgical Anatomy .................................................... 231

Surgical Technique ............................................. 232

Results ........................................................ 235

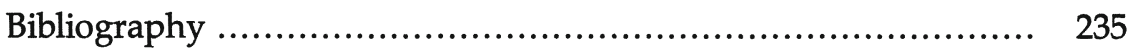

20 Ventricular Septal Defects ....................................... 237

Indications for Surgery .............................................. 237 
Choice of Operation .............................................. 238

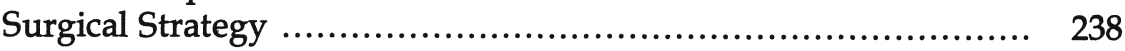

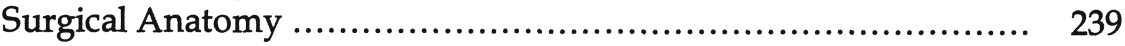

Surgical Technique .............................................. 241

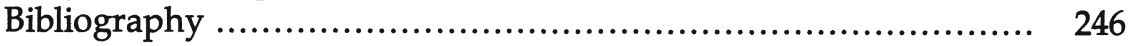

21 Tetralogy of Fallot ................................................ 249

Indications for Surgery ........................................... 249

Surgical Strategy ................................................. 250

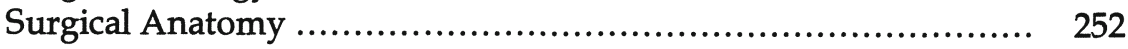

Surgical Technique .............................................. 254

Late Results .................................................... 258

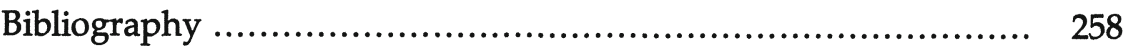

22 Transposition of the Great Arteries ............................. 261

Indications for Surgery ........................................... 261

Choice of Operation ............................................. 262

Surgical Strategy ................................................ 262

Arterial Switch Procedure .......................................... 264

Senning Operation ................................................ 270

Mustard Operation ............................................... 274

Bibliography .................................................... 279

23 Total Anomalous Pulmonary Venous Connection ......... 282

Indications for Surgery .......................................... 282

Surgical Strategy ................................................ 282

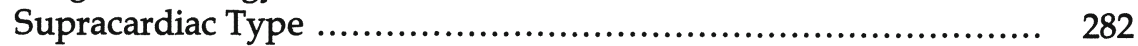

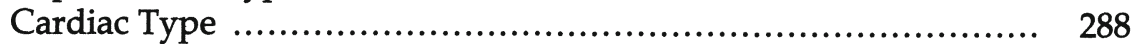

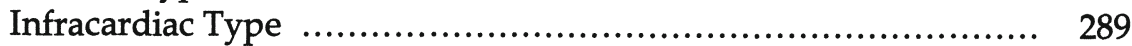

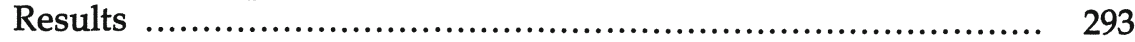

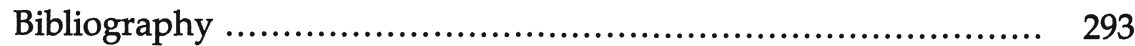

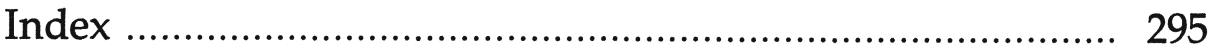

